CERN-EP/83-118

15 August 1983

\title{
HADRONIC JET PRODUCTION AT THE CERN PROTON-ANTIPROTON COLLIDER
}

\author{
UA1 Collaboration, CERN, Geneva, Switzerland
}

Aachen ${ }^{2}$-Annecy (LAPP) ${ }^{2}$-Birmingham ${ }^{3}-$ CERN $^{4}-$ Helsinki ${ }^{5}$-Queen Mary College, London ${ }^{6}-$ Paris (Coll. de France) ${ }^{7}$-Riverside ${ }^{8}-$ Rome $^{9}-$ Rutherford Appleton Lab. ${ }^{10}$ Saclay $(\mathrm{CEN})^{\mathrm{i1}-\text { Vienna }}{ }^{12}$ Collaboration

G. Arnison ${ }^{10}$, A. Astbury ${ }^{10}$, B. Aubert ${ }^{2}$, C. Bacci ${ }^{9}$, G. Bauer*, A. Bézaguet ${ }^{4}$, R.K. Bock ${ }^{4}$, T.J.V. Bowcock ${ }^{6}$, M. Calvetti ${ }^{4}$, T. Carroll ${ }^{4}$, P. Catz ${ }^{2}$, P. Cennini ${ }^{4}$, S. Centro ${ }^{4}$, F. Ceradini ${ }^{4,9}$, S. Cittolin ${ }^{4}$, D. Cline*, C. Cochet ${ }^{11}$, J. Colas ${ }^{2}$, M. Corden ${ }^{3}$, D. Dallman ${ }^{4,12}$, M. DeBeer ${ }^{11}$, M. Della Negra ${ }^{2,4}$, M. Demoulin ${ }^{4}$, D. Denegri ${ }^{11}$, A. Di Ciaccio ${ }^{9}$, D. DiBitonto ${ }^{4}$, L. Dobrzynski ${ }^{7}$, J.D. Dowell ${ }^{3}$, K. Eggert ${ }^{1}$, E. Eisenhandler ${ }^{6}$, N. Ellis ${ }^{4}$, P. Erhard ${ }^{1}$, H. Faissner ${ }^{1}$, G. Fontaine ${ }^{7}$, R. Frey ${ }^{8}$, R. Frühwirth ${ }^{12}$, J. Garvey ${ }^{3}$, S. Geer ${ }^{7}$, C. Ghesquière ${ }^{7}$, P. Ghez ${ }^{2}$, K.L. Giboni ${ }^{1}$, W.R. Gibson ${ }^{6}$, Y. Giraud-Héraud ${ }^{7}$, A. Givernaud ${ }^{11}$, A. Gonidec $^{2}$, G. Grayer ${ }^{10}$, P. Gutierrez ${ }^{8}$,

T. Hansl-Kozanecka ${ }^{1}$, W.J. Haynes ${ }^{10}$, L.O. Hertzberger**, C. Hodges ${ }^{8}$, D. Hoffmann ${ }^{1}$, H. Hoffmann ${ }^{4}$, D.J. Holthuizen**, R.J. Homer ${ }^{3}$, A. Honma ${ }^{6}$, W. Jank ${ }^{4}$, G. Jorat ${ }^{4}$, P.I.P. Kalmus ${ }^{6}$, V. Karimäki ${ }^{5}$, R. Keeler ${ }^{6}$, I. Kenyon ${ }^{3}$, A. Kernan ${ }^{8}$, R. Kinnunen ${ }^{5}$, H. Kowalski ${ }^{4}$, W. Kozanecki ${ }^{8}$, D. Kryn ${ }^{4,7}$, P. Kyberd ${ }^{6}$, F. Lacava ${ }^{4}$, J.-P. Laugier ${ }^{11}$, J.-P. Lees ${ }^{2}$, H. Lehmann ${ }^{1}$, R. Leuchs ${ }^{1}$, A. Lévêque ${ }^{11,4}$, D. Linglin ${ }^{2}$, E. Locci ${ }^{11}$, M. Loret ${ }^{11}$, J.-J. Malosse ${ }^{11}$, T. Markiewicz ${ }^{4}$, G. Maurin ${ }^{4}$, T. McMahon ${ }^{3}$, J.-P. Mendiburu' ${ }^{7}$, M.-N. Minard ${ }^{2}$, M. Moricca ${ }^{9}$, H. Muirhead***, F. Muller ${ }^{4}$, A.K. Nandi ${ }^{10}$, L. Naumann ${ }^{4}$, A. Norton ${ }^{4}$, A. Orkin-Lecourtois ${ }^{7}$, L. Paoluzi ${ }^{9}$, G. Piano Mortari, E. Pietarinen ${ }^{5}$, M. Pimiä ${ }^{5}$, A. Placci ${ }^{4}$, E. Radermacher ${ }^{1}$, J. Ransdell ${ }^{8}$, H. Reithler ${ }^{1}$, J.-P. Revol, J. Rich ${ }^{11}$, M. Rijssenbeek ${ }^{4}$, C. Roberts ${ }^{10}$, J. Rohlff ${ }^{4}$, P. Rossi ${ }^{4}$, C. Rubbia ${ }^{4}$, B. Sadoulet ${ }^{4}$, G. Sajot ${ }^{7}$, G. Salvi ${ }^{6}$, G. Salvini ${ }^{9}$, J. Sass $^{11}$, J. Saudraix ${ }^{11}$, A. Savoy-Navarro ${ }^{11}$, D. Schinzel ${ }^{4}$, W. Scott ${ }^{10}$, T.P. Shah ${ }^{10}$, D. Smith ${ }^{8}$, M. Spiro ${ }^{11}$, J. Strauss ${ }^{12}$, K. Sumorok ${ }^{4}$, F. Szoncso ${ }^{12}$, C. Tao**,

G. Thompson ${ }^{6}$, J. Timmer ${ }^{4}$, E. Tscheslog ${ }^{1}$, J. Tuominiemi ${ }^{5}$, J.-P. Vialle ${ }^{4}$, J. Vrana ${ }^{7}$, V. Vuillemin ${ }^{4}$, H.D. Wahl ${ }^{12}$, P. Watkins ${ }^{3}$, J. Wilson ${ }^{3}$, Y.G. Xie ${ }^{4}$, M. Yvert ${ }^{2}$, E. Zurfluh ${ }^{4}$

(Submitted to Physics Letters B)

*) University of Wisconsin, Madison, Wisconsin, USA.

**) NIKHEF, Amsterdam, The Netherlands.

***) Visitor from the University of Liverpool, England. 


\begin{abstract}
We present a detailed study of hadronic jets obtained in a data sample taken in the UA1 detector with a localized transverse energy trigger. We discuss the average shape of jets in terms of energy and charged particle content, and compare this to data generated in Monte Carlo programs. We further extend the previously reported inclusive jet cross section to the region of $\mathrm{E}_{\mathrm{T}}=100 \mathrm{GeV}$. A comparison with theoretical models of cross sections for events with more than two jets is also given.
\end{abstract}

\title{
1. Introduction
}

High transverse energy depositions in calorimeters of large aperture have been used previously to demonstrate the existence of hadronic jet events in hadron-hadron collisions when the energy in the centre of mass, $V s$, is sufficiently high $[1,2,3]$. It was also found that the efficiency of transverse energy triggers in tagging hadronic jets depends strongly on the solid angle used to sum transverse energy depositions [5].

The UA1 detector at the CERN proton-antiproton collider has the unique feature of covering almost the full solid angle [4] whilst operating at the highest energy available today, at $v_{s}=540 \mathrm{GeV}$.

In the first exposure on the collider in 1981, we used a total transverse energy $\left(\Sigma\left|E_{T}\right|\right)$ trigger over the full pseudorapidity range (pseudorapidity $\eta \equiv-\log [\tan (\Theta / 2)])$ of our central calorimeters $(|\eta|<$ 3) as an unbiased way to search for jets. Jets were shown to dominate over high multiplicity events for $\Sigma\left|E_{T}\right|>100 \mathrm{GeV}$, when restricting the sum to a smaller solid angle $|\eta|<1.5$.

With a small data sample, the jet yield measured in the 1981 exposure was found to be in agreement with QCD expectations. We have now recorded a thousand times more integrated luminosity using a localized transverse energy trigger rather than a global $\Sigma\left|E_{T}\right|$ trigger. 
In this letter, we present a detailed investigation of the shape and the production rates of hadronic jets at large $E_{T}$. Jet fragmentation into charged particles and angular distributions of two-jet systems are discussed in separate publications $[6,7]$.

\section{Apparatus}

The components of the UA1 detector relevant for measuring jets, and the energy response and calibration of calorimeters have been previously described [2]. We recall briefly the four main parts of the central calorimetry, two electromagnetic calorimeters called Gondolas and Bouchons, surrounded by two hadronic calorimeters called $\mathrm{C}-\mathrm{s}$ and $\mathrm{I}-\mathrm{s}$. Their coverage in solid angle and their segmentation are given in Table 1. The Gondolas are not segmented in azimuth. The azimuthal angle of the energy centroid is determined by the ratio of the light pulses recorded at each of the four corners of each Gondola cell. In the Bouchon, proportional tubes installed after 11 radiation lengths determine the shower centre in the plane perpendicular to the beam.

The Central Detector [4], a drift chamber volume $5.8 \mathrm{~m}$ long and $2.3 \mathrm{~m}$ in diameter, gives an accurate 3-dimensional picture of all charged tracks in the event, and allows the measurement of charged track momenta in a horizontal magnetic field of $0.7 \mathrm{~T}$.

\section{Event Selection}

\subsection{Data Taking}

The data presented here come from an exposure with an integrated luminosity of 14 events/nb, recorded between October and December 1982. A collision is defined by a pretrigger hodoscope requiring tracks on both sides of the interaction point in the angular range from 12 to 300 or 5 to 9 mrads. The hodoscope part covering the region from 12 to 56 mrads is used for monitoring luminosity, with the absolute scale set by assigning a cross section of 35 mbarn to this range.

An inclusive jet trigger is then defined by a fast hardware processor which further requires a localized transverse energy deposition in 
excess of $15 \mathrm{GeV}$ in a window of $2 \mathrm{C}$ calorimeters summed with the 8 corresponding Gondola cells, covering an aperture of roughly $\Delta \eta=$ 0.75, $\Delta \phi=\pi$ (see fig. 1). This hardware trigger was subsequently redefined in an off-line filtering program to select events using the same calorimetric window with a threshold set at $25 \mathrm{GeV}$. Time-of-flight information from the pretrigger hodoscope was used to discard noncollision triggers $(\approx 5 \%)$,

\subsection{Jet Definition}

Jets are defined as clusters in pseudorapidity/azimuth $(\eta / \phi)$ space by the following procedure [2]. An energy vector is associated to each calorimeter cell. For hadronic cells, the vector points from the interaction vertex to the centre of the cell. For electromagnetic cells, the vector points to the energy centroid determined by pulse height measurements (Gondolas) or by position detectors (Bouchons).

In the subsequent clustering, cells are treated differently depending on their $E_{T}$ being above or below $2.5 \mathrm{GeV}$ :

- Among the cells with $E_{T} \geq 2.5 \mathrm{GeV}$, the highest $\mathrm{E}_{\mathrm{T}}$ cell initiates the first jet. Subsequent cells are considered in order of decreasing $E_{T}$. Each cell in turn is added vectorially to the jet closest in $(\eta, \phi)$ space, i.e. with the smallest $d \equiv V\left(\Delta \eta^{2}+\Delta \phi^{2}\right)$ (with $\phi$ in radians), if $d \leq 1.0$. If there is no jet with $d \leq 1.0$, the cell initiates a new jet.

- Cells with $E_{T}<2.5 \mathrm{GeV}$ are finally added vectorially to the jet nearest in $(n, \phi)$ if their transverse momentum relative to the jet axis is less than $1 \mathrm{GeV}$ and if they are not further than $45^{\circ}$ in direction from the jet axis.

The cut at $d=1.0$ has been derived from the jet energy profile (see below).

\subsection{Off-line Cuts and Trigger Jets}

All triggers selected previously were fully reconstructed in the central detector, and calorimeter depositions were corrected using detailed light attenuation maps and an average response correction factor of 1.13 to allow for the hadronic energy in the electromagnetic calorimeters [2]. After reconstruction of jets according to the procedure above over the 
full pseudorapidity range $|\eta|<3$, an $E_{T}$ threshold was applied requiring at least one jet with $\mathrm{E}_{\mathrm{T}}>30 \mathrm{GeV}$ in the Gondola region. This jet is called the trigger jet. A threshold was applied to the fraction of jet energy deposited in the electromagnetic calorimeters $(\geq 10 \%)$, to discard signals from beam halo in the hadronic calorimeters. This leaves $<1 \%$ of events with a potential halo problem, while reducing the good data sample by $<2 \%$. To avoid edge effects due to the calorimeter geometry, the trigger jet axis has to be contained in $\eta= \pm 1.2$ and in $\phi$ $= \pm 60^{\circ}$ from the horizontal plane. These cuts ensure good energy containment and minimal particle losses due to the narrow dead zones between the calorimeters in the vertical plane.

To understand possible trigger biases, we used a sample of events taken with a global (i.e. $|\eta|\langle 1.5) \Sigma\left|E_{T}\right|>20 \mathrm{GeV}$ trigger and studied the efficiency of jet finding. We found that the localized energy trigger and the selection procedure is $\approx 80 \%$ efficient for jets with $E_{T}=30 \mathrm{GeV}$, and more than $95 \%$ efficient for jets with $E_{T} \geq 35 \mathrm{GeV}$.

\section{The Shape of Jets}

\subsection{Determination of Jet Axis and Jet Energy.}

The above jet finding procedure results in jets with energy and axis defined from calorimeter depositions. It is therefore necessary to discuss the precision of these parameters.

The definition of a jet axis is rather independent of the algorithm used for finding the jet, as it is mostly dependent on large energy depositions in few calorimeter cells. To assess the precision of the jet axis we have simulated in our detector high $E_{T}$ jets using a Monte Carlo program with fragmentation according to a cylindrical phase space (CPS) model defined below. The result of this study is that the jet axis given by the jet finding procedure agrees with the vectorial sum of the momenta of fragments from the generated jet to within $\pm 6^{\circ}$ in $\phi$ and \pm 0.04 in $n$ ( $\mathrm{rms}$ ). This shows that the granularity of our calorimeters does not introduce any appreciable error in the jet axis definition. If using the same jet finding algorithm on the charged tracks given by the central detector, determined with superior angular resolution, one 
obtains a charged jet axis which coincides with the calorimetric jet axis to within \pm 0.1 in $\eta$ and $\pm 10^{\circ}$ in $\phi$ ( $\mathrm{rms}$ ). This difference reflects mostly the fluctuations between the charged and neutral parts of jets, and constitutes a lower limit to the precision of the jet axis definition.

The definition of the jet energy, on the other hand, is directly related to the cutoff parameter $d$ in $(\eta, \phi)$ space. We use the energy profile and Monte Carlo studies to obtain better understanding of the jet energy.

\subsection{Energy and Charged Particle Momentum Profiles}

Given the axis of a jet, the average values per jet of deposited transverse energy and of charged particle transverse momentum can be studied as function of $\Delta \eta$ and $\Delta \phi$ referred to the jet axis. We restrict ourselves here to the pseudorapidity projection, where the granularity is best. We define an average jet profile by superimposing many jets, leaving out from the average any low-acceptance regions in $\eta$ or $\phi$. The hemisphere opposite to the jet axis in $\phi(|\Delta \phi|>\pi / 2)$ is not included. All jets found in our event sample are included, if their transverse energy is at least $20 \mathrm{GeV}$ and if their axis lies within the same $(\eta, \phi)$ limits as used for the trigger jet.

The transverse energy flow as a function of $\Delta \eta$ is shown in fig.2 (a-c) for three ranges of jet $E_{T}$. A clear enhancement is observed on top of a flat energy plateau. The full width of the enhancement at the base is given by $\Delta \eta= \pm 1.0$, independent of the jet energy (this justifies the cutoff value $d \leq 1.0$ in the jet finding algorithm).

We distinguish two distinct regions in the average jet, the hard core of the jet $(|\Delta \eta|<0.2)$, and the wings $(0.2 \leq|\Delta \eta|<1.0)$. The relative amount of transverse energy contained in the core increases with the jet $E_{T}$. The $E_{T}$ content of the wings seems rather independent of the jet $E_{T}$. Outside the enhancement, for $|\Delta \eta|>1.0$, a constant $E_{T}$ plateau is observed, whose height is independent of the jet $E_{T}$. Its value is substantially higher than the one observed for minimum bias events [11]. When the events with more than two jets are excluded the plateau height lies between the values for minimum bias events and for all events. Hence the origin of the plateau is in two distinct phenomena. The first is due to the debris of the beam particles, namely spectator 
jets. The second one is the production of multijet events, clearly observed in the present data sample. In this case more than one jet can be emitted in the same azimuthal hemisphere, thus increasing the height of the plateau. It should be noted, though, that an absolute measurement of this plateau is difficult: the particles outside the hard core of the jet are mostly of low momentum, and are not measured with good precision due to the non-linear response of the calorimeters at low energy [14].

We have therefore used the information coming from the central detector for a more quantitative assessment of the jet and plateau composition. The jet profile is now studied with respect to the charged jet axis. Again, particle losses in the small regions of low acceptance in the central detector are corrected for by our averaging procedure.

Figs. $2(d-f)$ show the mean charged transverse momentum flow in the same slices of jet $\mathrm{E}_{\mathrm{T}}$ as before. The same conclusions are reached as for the transverse energy flow. This demonstrates in particular that the granularity of our calorimeters does not influence our measurement of the jet width. The distributions also show that calorimetric measurements for energetic jets are not significantly distorted by the magnetic field.

\subsection{Multiplicity Profile}

The multiplicity flow is obtained by the same averaging procedure as the energy flow, counting tracks reconstructed in the central detector and excluding regions of low acceptance. In figs. $2(\mathrm{~g}-\mathrm{i})$ the average multiplicity flow of charged tracks araound the charged jet axis is shown, again as function of the jet $E_{T}$. The multiplicity in the jet increases only slowly with $E_{T}$, implying that the leading particles of the jet carry an increasing transverse momentum.

Outside the jet a flat plateau is observed. The mean multiplicity for the plateau depends on the topology of the events. A definite increase in the average multiplicity is observed over the minimum bias level [8]. For pure 2-jet events the rise is $40 \%$, and is almost a factor of 2 when the multijet events are included.

A similar increase is observed in the mean $\mathrm{p}_{T}$ per charged particle over the minimum bias value, $14 \%$ for 2 -jet events and $25 \%$ when including the multijet events. 
The increase observed in the transverse energy flow plateau over minimum bias events can therefore be attributed to an increase of both the average multiplicity and the mean $\mathrm{p}_{T}$ per particle, due to the multijet production.

The precise determination of charged particle multiplicities inside the jets is difficult. It is not possible to separate low-momentum fragments of the jet which contribute sizeably to the jet multiplicity, from the multiplicity background arising from spectator or additional jets, on an event-by-event basis. To estimate losses of low-momentum fragments from the jet by the jet finding algorithm, we rely upon a fragmentation model and a full Monte Carlo simulation of the detector.

\subsection{Monte Carlo Simulation}

Two Monte Carlo programs with different hadronization models have been compared with the data. The first one is a naive parton-parton hard scattering model without QCD radiative effects, in which the systems of hard scattered partons and spectator jets fragment independently according to cylindrical phase space (CPS). The mean multiplicity of fragments is obtained as a function of $s$ (square of c.m.s. parton-parton energy) from a phenomenological fit to multiplicity measurements in hadronic events from $\mathrm{e}^{+} \mathrm{e}^{-}$collisions [10]:

$$
\langle n(s)\rangle=2.0+0.027 \exp \left\{2 v\left[\ln \left(s / \wedge^{2}\right)\right]\right\}
$$

with $\wedge=0.3 \mathrm{GeV}$.

The second program is ISAJET [12], which incorporates QCD radiated gluons in the final state. The fragmentation model used for the spectators in ISAJET is tuned to reproduce minimum bias events.

For both models, generated particles are tracked through the magnetic field and the showers in the calorimeters are simulated [13].

In fig. 3 the experimental transverse energy flow for jets with $E_{T}>35$ $\mathrm{GeV}$ is compared to the Monte Carlo results. ISAJET gives a better description of the jet shape than CPS. Both programs fail to reproduce the plateau region as they do not include a complete multijet production, in particular through initial state bremsstrahlung. The charged multiplicity flow is shown in fig. 4, again for jets with $E_{T}>35$ $\mathrm{GeV}$. The multiplicity given by ISAJET is low and coincides with the 
minimum bias level. CPS gives a good overall description of the multiplicity flow.

To measure charged jet multiplicities, we sum up average track multiplicities in the window $\Delta \eta= \pm 1$, apply a flat background subtraction obtained from the region $1 \leq|\Delta \eta|<2$, and correct for the loss of jetassociated particles outside $\Delta \eta= \pm 1$ according to CPS. As in our data, low-acceptance regions are excluded and corrected for. A further correction is applied for unreconstructed low-momentum particles. The final corrected results on charged track multiplicities in jets are shown in table 2.

It should be noted that ISAJET when tuned to fit the plateau multiplicity results in larger corrections to the multiplicity inside the jet window, as more fragments are generated far from the jet axis. The $10 \%$ systematic error in table 2 is an attempt to account for this model dependence of the correction.

Using the multiplicity extrapolation (1) from $e^{+} e^{-}$for our energy range would also result in higher multiplicities, of the order of 9.0 charged particles for a jet of $25 \mathrm{GeV}$. A more detailed comparison with $\mathrm{e}^{+} \mathrm{e}^{-}$jets can be found in the discussion of jet fragmentation [6].

\section{Inclusive Jet Cross Section}

To measure the inclusive jet yield, all events are selected as above, imposing additional constraints on the vertex position $( \pm 40 \mathrm{~cm}$ from the detector's centre, for a measured rms spread of $\pm 12 \mathrm{~cm}$ ) and on the jet axes $\left( \pm 1\right.$ in pseudorapidity, $\pm 60^{\circ}$ in $\phi$ from the horizontal plane). All jets with $E_{T}>35 \mathrm{GeV}$ are considered. Events containing jets with transverse energy above $55 \mathrm{GeV}$ were, in addition, inspected visually on a MEGATEK 3-dimensional display, and a small number of badly reconstructed or background events were rejected, typically events in which cosmic showers or beam halo overlap with collisions.

The inclusive $E_{T}$ distribution thus obtained is further corrected for geometrical acceptance, experimental procedure and detector resolution using a Monte Carlo method. Jets are simulated using the models 
discussed above. The comparison between the reconstructed $E_{T}$ spectrum and the Monte Carlo input results in a correction factor for each bin of $E_{T}$. The global correction is not strongly dependent on $E_{T}$ and does not exceed a factor 1.25.

The inclusive cross section $d \sigma / d_{T} d \eta$ obtained after correction is shown in fig.5, together with the data from 1981 published in [2]. In the small region of overlap the measurements agree. All errors shown are purely statistical. The following systematic errors are not included:

- The uncertainty of the absolute luminosity $( \pm 15 \%)$;

- Uncertainties in geometrical acceptance due to the choice of the fiducial region, of the jet finding algorithm and of the fragmentation model used in the Monte Carlo programs ( $\pm 5 \%$ );

- An uncertainty in the jet energy which for a given event varies when changing the angular aperture in the jet finding algorithm. This translates into a $\pm 20 \%$ uncertainty in cross section;

- The dependence of the jet acceptance and jet finding efficiency on the production and fragmentation models used in the Monte Carlo programs $( \pm 20 \%)$;

- The uncertainty arising from the definition of the energy scale: The absolute energy calibration is known to \pm 5 \% [2].

- The additional uncertainty in energy scale due to the poorly known particle composition of jets: Electromagnetic and hadronic calorimeters exhibit a different response to electromagnetic particles and hadrons, and this response difference is strongly dependent on the particle momenta for $p<5 \mathrm{GeV}$ [14]. The uncertainty in the average response correction used is estimated at $\pm 5 \%$. We combine these energy effects into an overall error of $\pm 7.5 \%$ of the energy scale, which translates into an uncertainty of a factor 1.5 in the cross section.

Our overall systematic uncertainty in the cross section can therefore be given as a factor of 1.65 , essentially independent of the jet transverse energy.

The measured cross section is compared in the figure to a band of QCD predictions with a width corresponding to uncertainties in the theory [15]. Note that QCD predicts transverse momentum distributions for 
partons, while our measurements refer to the sum of the jet fragment momenta. Despite large experimental and theoretical uncertainties, the agreement observed is excellent. The large increase in jet cross section from ISR [3] to collider [1,2] energies, by three orders of magnitude, is thus confirmed.

\section{Multijet Production}

In order to obtain the cross sections for producing several jets in an event, we now consider, in addition to the trigger jet, all jets with $\mathrm{E}_{T}$ $>15 \mathrm{GeV}$ reconstructed with a jet axis inside $|\eta|<2.5$, again attempting to avoid edge effects and jets that might be faked by spectator background.

In fig. 6 we show the fraction of events with 1,2 and 3 jets (trigger jets included) as function of the $E_{T}$ of the trigger jet. The 2-jet topology dominates over the full range in $E_{T}$ at a level of $\approx 80-85 \%$. The fraction of 1 -jet events becomes negligible at high trigger jet $E_{T}$, whereas the fraction of 3-jet events rises in the region of low $E_{T}$ and levels off at $\approx 15 \%$.

We should stress that our jet finding algorithm, with the window $\Delta \eta=$ \pm 1 , and the additional requirement $E_{T}>15 \mathrm{GeV}$, can be expected to have a direct influence on the number of jets found, and that the topological cross sections as presented here have to be understood in relation to a given jet finding procedure. We also have not corrected these cross sections in any way for geometrical acceptance.

The occasional presence of a third jet strongly suggests a gluon bremsstrahlung mechanism similar to what has been observed in hadronic $e^{+} e^{-}$events. QCD predicts multijet events due to quark-gluon and gluon-gluon couplings with rates that are proportional to the products of coupling constants appearing in the bremsstrahlung processes of the original parton. 3-jet events would then, for instance, be produced with a cross section roughly $\alpha_{s}$ times the cross section for 2 -jet production.

The rate of multijet events can be estimated by measuring the differential cross section in terms of some suitable parameter describing 
the non-coplanarity. One such parameter is $p^{\text {out }}$, the momentum perpendicular to the plane defined by the trigger jet and the beam momentum. For large enough $p^{\text {out }}$ the 3 -jet production rate can be calculated perturbatively from $\operatorname{QCD}[15,16]$ and be compared with the data.

For the study of non-coplanarity we used events with a trigger jet as defined in 3.3 above. In order to minimize any effects coming from problems in jet finding we first calculate $p^{\text {out }}$ directly from all $E_{T^{-}}$ vectors not belonging to the trigger jet. To avoid contamination from the spectator jets we require these $\mathrm{E}_{T}$-vectors to have $|\eta|<2.5$. The $p^{\text {out }}$ is reconstructed by adding the $E_{T}$-vector components perpendicular to the plane defined by the energy axis of the trigger jet and the beam direction, separately on both sides of the plane. If the energies of the jets are balanced, the two $\left|p^{\text {out }}\right|$ values should be the same. The difference shows a width which is consistent with the experimental resolution. We therefore take as $p^{\text {out }}$ the average value of $\left|p^{\text {out }}\right|_{\text {left }}$ and $\left|p^{\text {out }}\right|_{\text {right }}$.

The resulting $p^{\text {out }}$ distribution is shown in fig. 7a. This distribution has a contribution from the $\mathrm{p}_{\mathrm{T}}$ of the background, i.e. from particles not belonging to the jets, which is difficult to estimate accurately. From the transverse energy flow in minimum bias events one can roughly estimate the contribution to be about $5 \mathrm{GeV}$. To understand further the size of the background we have repeated the analysis using the jets as found by the jet algorithm. The results are shown in fig $7 b$. One sees that the $p^{\text {out }}$ distributions obtained have the same slope but are shifted by about $5 \mathrm{GeV}$ down in $\mathrm{p}^{\text {out }}$ when compared with the distribution obtained from all $E_{T}$-vectors. This shift is consistent with the estimate from minimum bias events. In the same figure is also shown the $p^{\text {out }}$ distributions obtained from events with two back-toback jets generated with our CPS Monte Carlo. In this model the partons are strictly coplanar, the non-zero pout comes from the hadronization model and the resolution of the apparatus. It is clear from the figure that this 2 -jet model cannot reproduce the observed large pout tail.

Instead the distribution obtained from a complete QCD calculation for 3 -jet production [16] as shown in fig.7b agrees much better with the 
data. The band reflects again the theoretical uncertainties, which are of the same origin as for the inclusive jet cross section. Note that the QCD calculation is for partons, i.e. there is no broadening due to fragmentation and no background from spectator partons either. We conclude from our study that we observe multijet events with a rate that is roughly consistent with the expectation from QCD.

\section{Acknowledgements}

We gratefully acknowledge the help of the managements and the technical staff of CERN and all outside institutions collaborating in UA1. The following funding agencies in our home countries have contributed to this programme:

Fonds zur Foerderung der Wissenschaftlichen Forschung, Austria.

Valtion Luonnontieteellinen Toimikunta, Finland.

Institut National de Physique Nucleaire et de Physique de Particules, and Institut de Recherche Fondamentale (CEA), France.

Bundesministerium fuer Forschung und Technologie, Germany.

Istituto Nazionale di Fisica Nucleare, Italy.

Science and Engineering Research Council, United Kingdom.

Department of Energy, United States of America.

Thanks are also due to the following people who have worked with the collaboration in the preparation and data collection on the runs described here:
F. Bernasconi,
F. Cataneo,
A. -M. Cnops,
L. Dumps,
M. Edwards,
J.-P. Fournier,
A. Micolon,
S. Palanque,
P.Queru,
P.Skimming,
G.Stefanini, M.Steuer, J.-C.Thevenin, H.Verweij and R.Wilson. 
References

[1] M.Banner et al., Phys. Lett. 118B (1982) 203.

[2] G.Arnison et al., Phys. Lett. 123B (1983) 115.

[3] T.Akesson et al., Phys.Lett. 118B (1982) 185 and 193.

T.Akesson et al., Phys. Lett. 123B (1983) 133.

[4] UA1 proposal, CERN/SPSC 78-06 (1978).

M. Barranco Luque et al., Nucl.Inst.Meth. 176 (1980) 175.

M.Calvetti et al., Nucl.Inst. Meth. 176 (1980) 255.

K. Eggert et al., Nucl.Inst. Meth. 176 (1980) 217 and 233.

A. Astbury, Physica Scripta 23 (1981) 397.

J.Timmer, Proceedings, 18th Rencontre de Moriond, 1983

(to be published).

[5] T.Akesson and H.Bengtson, Phys.Lett. 120B (1983) 133.

[6] Jet Fragmentation into Charged Particles at the CERN Proton-Antiproton Collider, UA1 collaboration, submitted to Phys. Lett.

[7] Angular Distributions and Structure Functions from 2-Jet Events at the CERN Proton-Antiproton Collider, UA1 collaboration, to be published.

[8] G.Arnison et al., Phys. Lett. 107B (1981) 320.

G.Arnison et al., Phys.Lett. 123B (1983) 108.

[9] G.Arnison et al., Phys. Lett. 118B (1982) 173.

[10] G.Wolf, DESY Report 81-086 (1981).

[11] Transverse Energy Distributions in the Central Calorimeters, CERN Internal Report EP/82-122 (1982).

[12] F.E.Paige and S.D.Protopopescu, ISAJET, BNL 31987.

[13] M.Della Negra, Physica Scripta 25 (1982) 468.

R.K.Bock et al., Nucl.Inst.Meth. 186 (1981) 533.

[14] M.J.Corden et al., Physica Scripta 25 (1982) 11.

C. Cochet et al., UA1 Tech. Note TN 82-40.

[15] Z. Kunszt and E.Pietarinen, CERN preprint TH 3584 (1983).

[16] Z.Kunszt and E.Pietarinen, Nucl.Phys.B164 (1980) 45.

T.Gottschalk and D.Sivers, Phys.Rev.D21 (1980) 102.

F.Berends et al., Phys.Lett. 103B (1981) 124. 


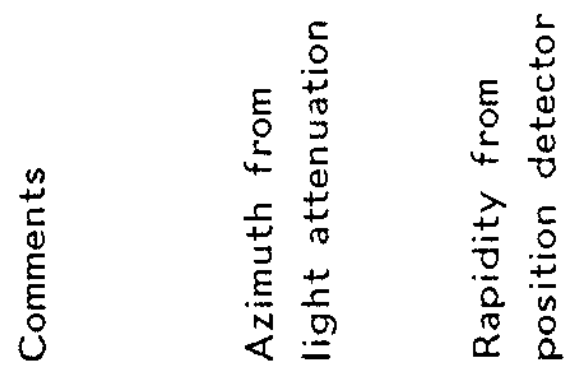

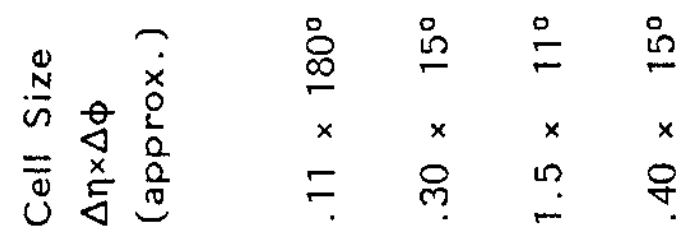

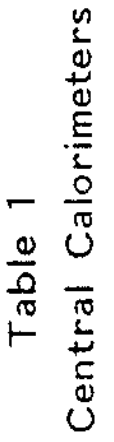

离

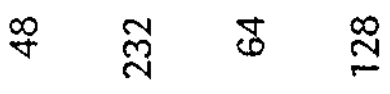

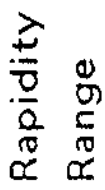

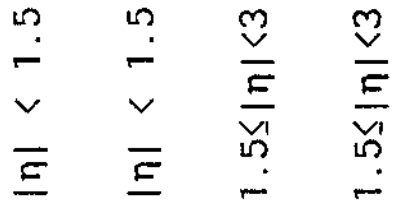

5

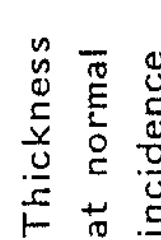

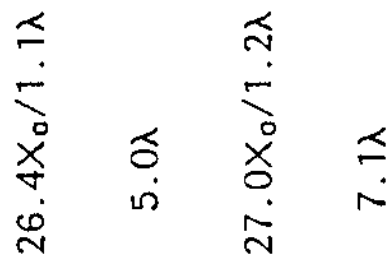

n
5
5
0
5
0

$\stackrel{\oplus}{\stackrel{0}{\nearrow}}$

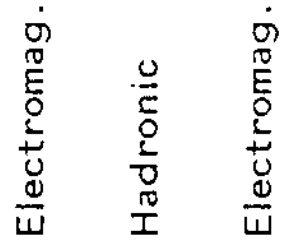

0
$\stackrel{0}{E}$
0
$\frac{1}{0}$
$I$
$I$

5
0
0
0
$\frac{1}{2}$
0
0
0
0
$<$
5
5
$\frac{5}{4}$
0
$\frac{1}{0}$
0

$\frac{1}{0}$

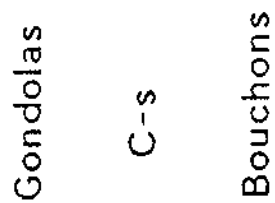

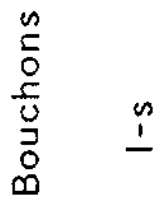

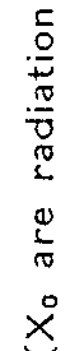


PAGE 15

Table 2

Charged Track Multiplicities in Single Jets

$\begin{array}{lccc}\mathrm{E}_{\mathrm{T}} \text { (Jet) } & 20-30 \mathrm{GeV} & 30-40 \mathrm{GeV} & >40 \mathrm{GeV} \\ \left\langle\mathrm{E}_{\mathrm{T}}\right\rangle \text { in bin } & 24.3 \mathrm{GeV} & 33.5 \mathrm{GeV} & 47.4 \mathrm{GeV} \\ \left\langle\mathrm{n}_{\text {charged }}\right\rangle & 6.7 \pm 0.7 & 7.5 \pm 0.7 & 7.7 \pm 0.8\end{array}$


Figure Captions

Fig. 1 : The UA1 Central Calorimeter, horizontal cut along the beam axis. Hatched areas represent a possible localized energy trigger configuration.

Fig. 2 (a-c): Transverse energy flow as function of $\Delta \eta$, i.e. pseudorapidity distance from the jet axis, for 3 slices of jet $E_{T}$. Cells inside $\Delta \phi= \pm 90^{\circ}$ are used.

(d-e): Charged transverse momentum flow as function of $\Delta \eta$.

$(\mathrm{g}-\mathrm{i})$ : Charged multiplicity flow as function of $\Delta \eta$.

Fig. 3 : Transverse energy flow for jets with $E_{T}>35 \mathrm{GeV}$ as function of $\Delta \eta$. Dashed curve: Cylindrical Phase Space model. Full curve: ISAJET model.

Fig. 4 : Charged multiplicity flow for jets with $E_{T}>35 \mathrm{GeV}$ as function of $\Delta \eta$. Dashed curve: Cylindrical Phase Space model. Full curve: ISAJET model.

Fig. 5 : Inclusive Jet cross section $d \sigma / d E_{T} d \eta(\eta=0)$ as function of $E_{T}$. 1981 data $(x)$ and 1982 data $(+)$. The hatched band corresponds to possible QCD predictions [15].

Fig. 6 : Fraction of events with 1,2 and 3 jets of $E_{T}>15 \mathrm{GeV}$, found by the jet algorithm in a pseudorapidity window $|\eta|<2.5$, as function of the trigger jet $\mathrm{E}_{\mathrm{T}}$.

Fig. 7 : pout distribution from calorimeter cells (a) and from jets (b), compared to a 2-jet model and to a perturbative QCD 3-jet calculation [16] . 


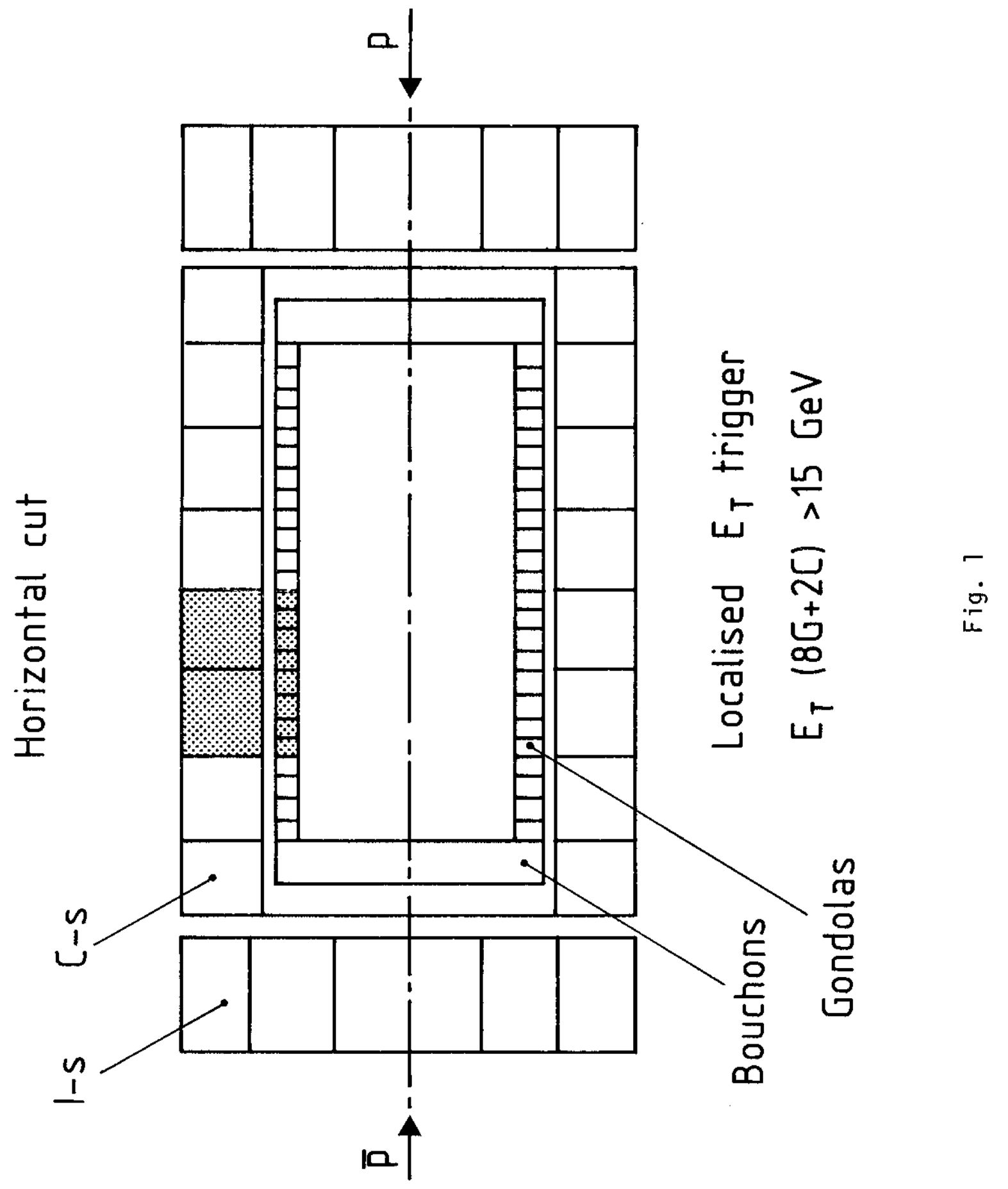



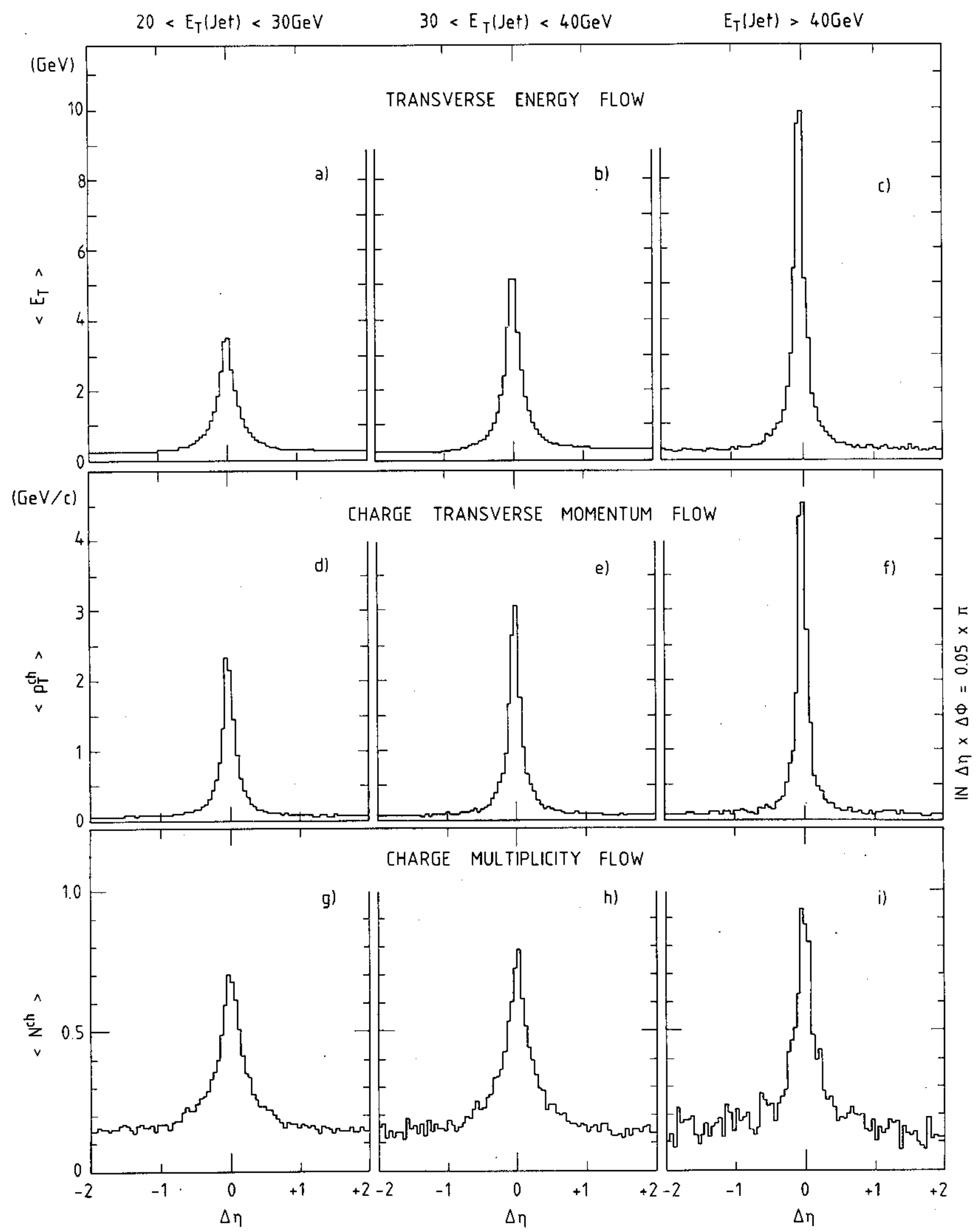

Fig. 2 


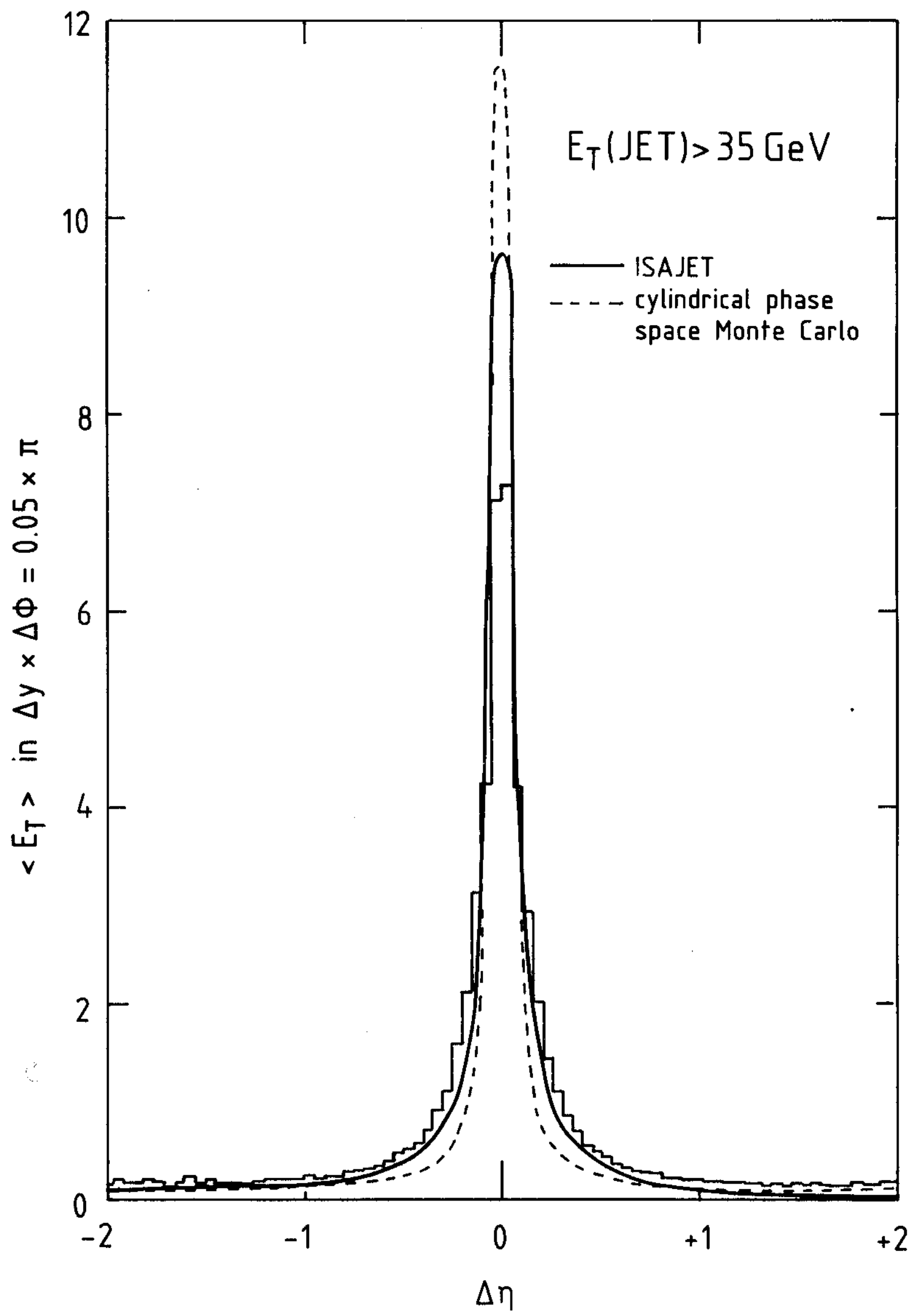

Fig. 3 


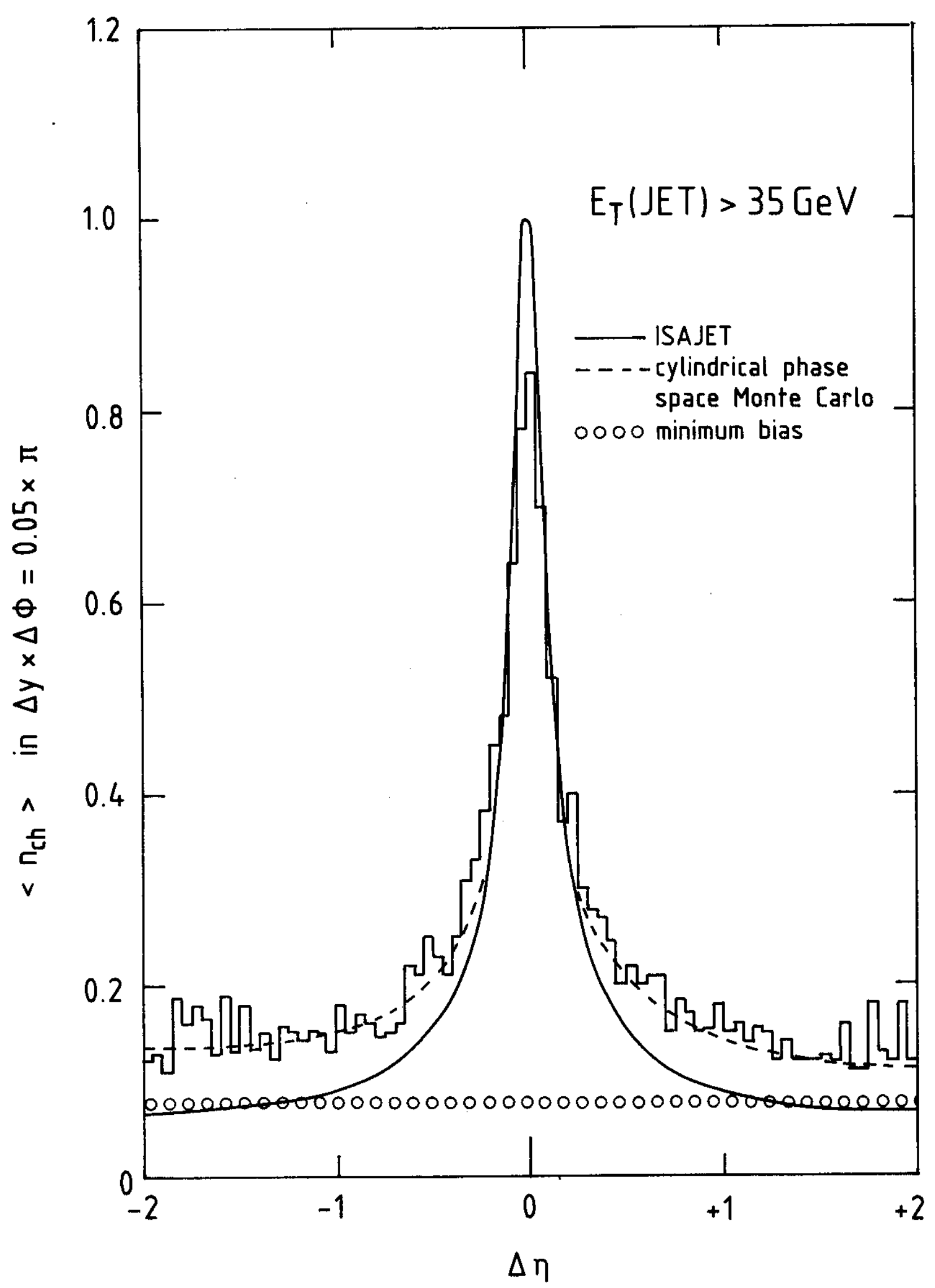

Fig. 4 


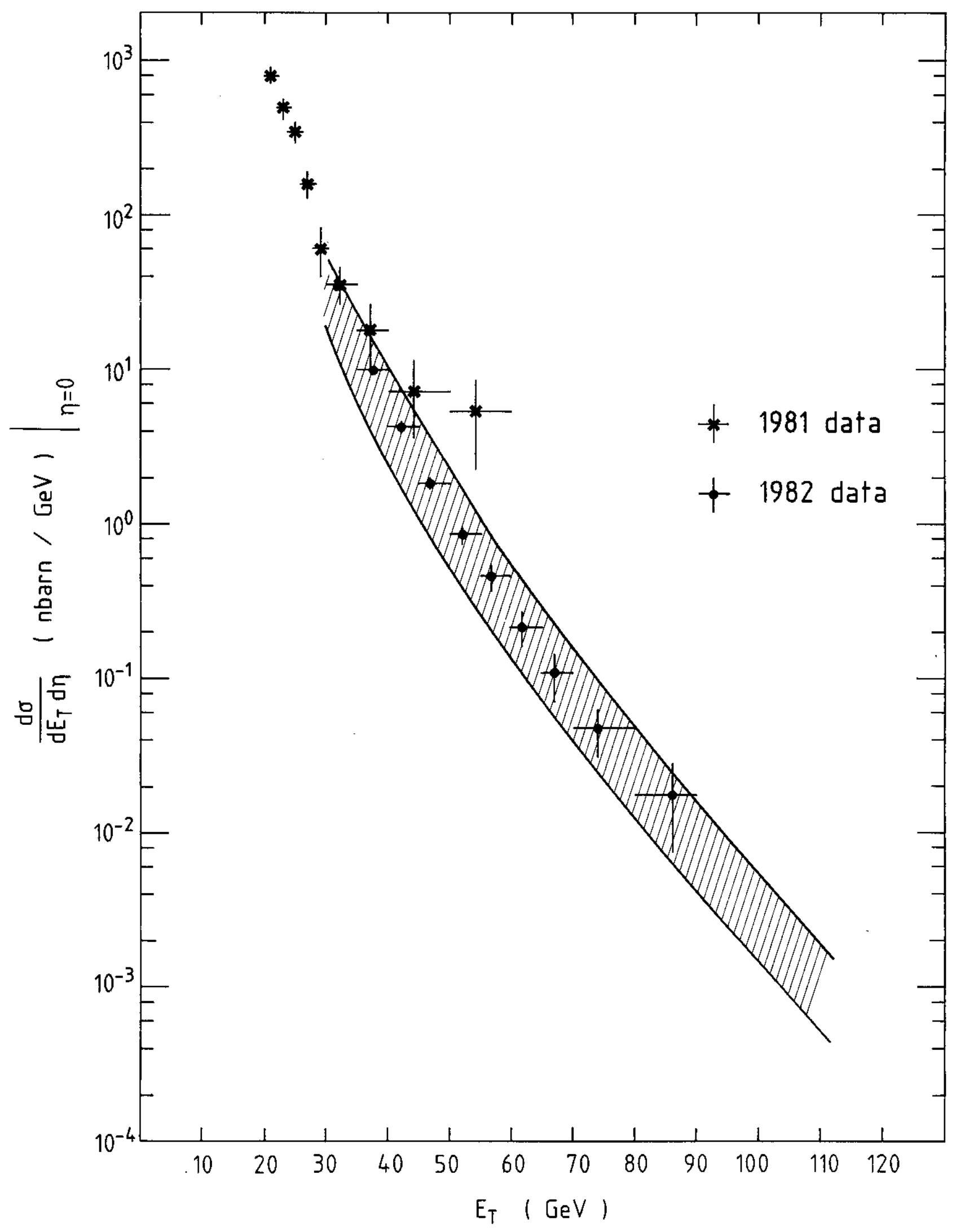

Fig. 5 


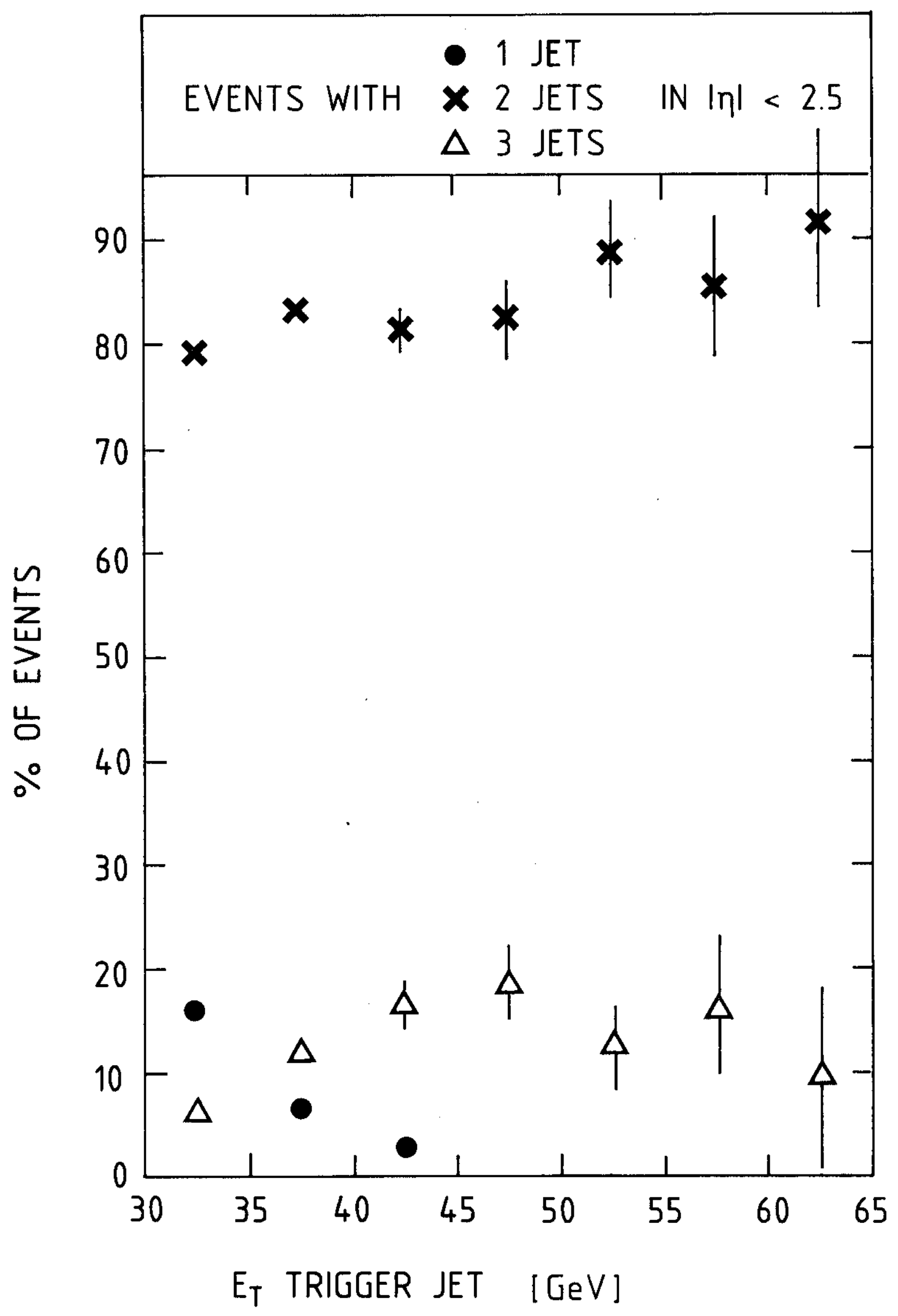

Fig. 6 


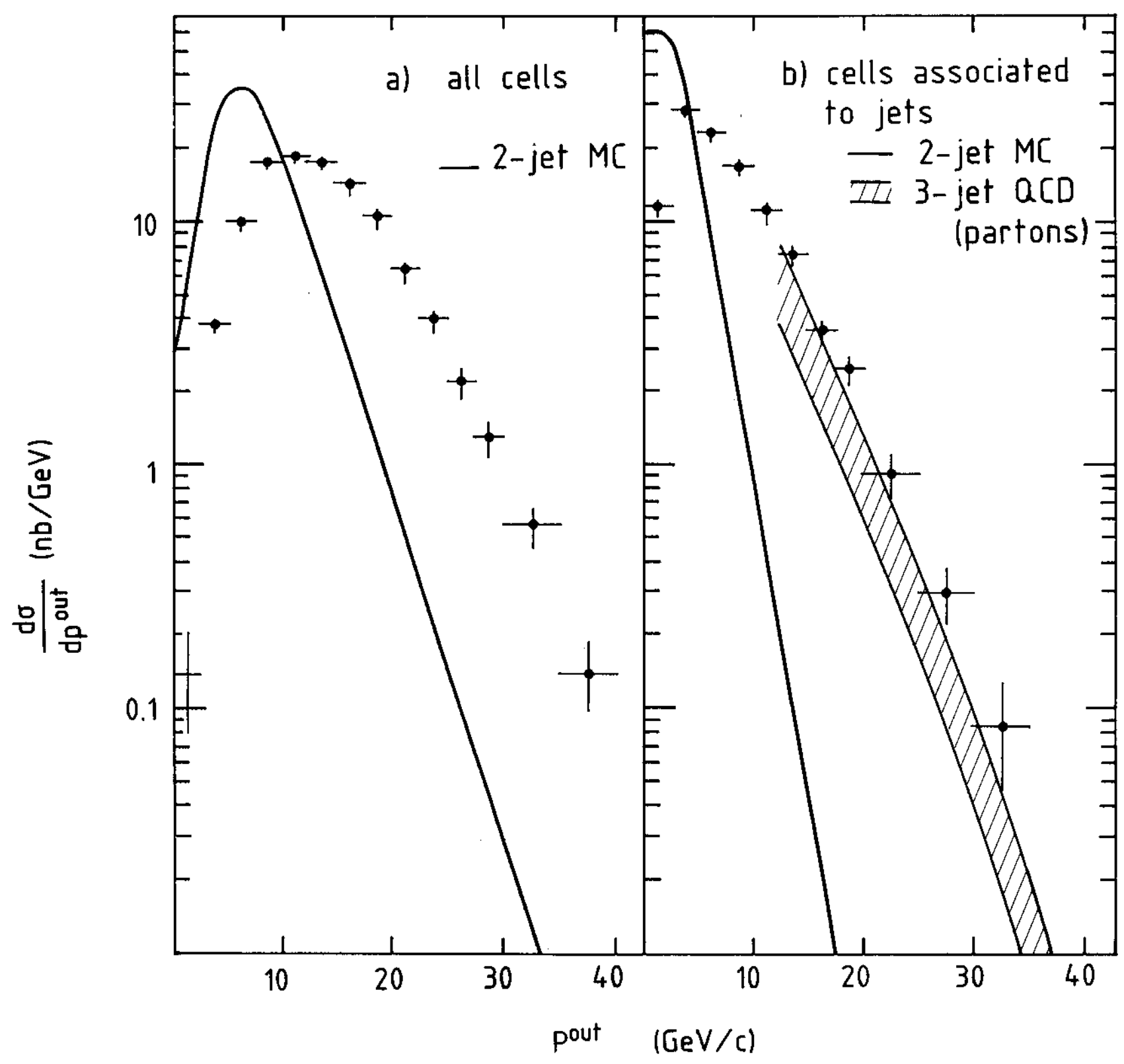

Fig. 7 


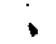

